\title{
CONIC-CONNECTED MANIFOLDS
}

\author{
PALTIN IONESCU* AND FRANCESCO RUSSO**
}

\begin{abstract}
We study a particular class of rationally connected manifolds, $X \subset$ $\mathbb{P}^{N}$, such that two general points $x, x^{\prime} \in X$ may be joined by a conic contained in $X$. We prove that these manifolds are Fano, with $b_{2} \leqslant 2$. Moreover, a precise classification is obtained for $b_{2}=2$. Complete intersections of high dimension with respect to their multi-degree provide examples for the case $b_{2}=1$. The proof of the classification result uses a general characterization of rationality, in terms of suitable covering families of rational curves.
\end{abstract}

A Gaetano Scorza che un secolo fa aveva colto l'importanza delle varietà razionalmente connesse considerando la classe particolare di varietà conicamente connesse formata da quelle di ultima specie.

"Invece per le $V_{4}$ di prima e terza specie arrivo a caratterizzarle tutte valendomi della teoria dei sistemi lineari sopra una varietà algebrica e, per le ultime, della circostanza che esse contengono un sistema $\infty^{6}$ di coniche così che per ogni loro coppia di punti passa una e una sola conica. Inoltre la natura dei ragionamenti è tale da mostrare come i risultati ottenuti possano estendersi, almeno per la maggior parte, alle varietà (di prima e ultima specie) a un numero qualunque di dimensioni, ...”, [Sc2, Opere Scelte, vol. 1, p. 253].

\section{INTRODUCTION}

A projective manifold $X$ is rationally connected if any two given (general) points of it may be joined by a rational curve. Since their introduction by Kollár, Miyaoka, and Mori [KMM], rationally connected manifolds were intensively studied, as they naturally generalize both classical notions of unirational and Fano manifold. When such an $X$ is embedded in some projective space, a measure of its complexity is given by the minimal degree of a family of rational curves possessing the above defining property. As a first instance we have linear spaces, characterized by the presence of a line through any two distinct points. The next case is what we call conic-connected manifolds: They are embedded manifolds $X \subset \mathbb{P}^{N}$ such that for any two general points $x, x^{\prime} \in X$, there is some irreducible conic joining $x, x^{\prime}$ and contained in $X$.

2000 Mathematics Subject Classification. 14M99, 14J45, 14E30.

Key words and phrases. Rationally connected, conic-connected, Fano manifold, quasi-line.

*Partially supported by the Italian Programme "Incentivazione alla mobilità di studiosi stranieri e italiani residenti all'estero".

**Partially supported by CNPq (Centro Nacional de Pesquisa), Grants 300961/2003-0, 308745/2006-0 and 474475/2006-9, and by PRONEX/FAPERJ-Algebra Comutativa e Geometria Algebrica. 
The main results of this paper, Theorem 2.2 and Corollary 2.3, show that, over $\mathbb{C}$, conic-connected manifolds are Fano, with second Betti number $b_{2} \leqslant 2$. Moreover, those with $b_{2}=2$ are completely described in the following short list: Segre products of two projective spaces and their hyperplane sections, or the inner projections, from a linear space, of the Veronese variety $v_{2}\left(\mathbb{P}^{n}\right)$. When $b_{2}=1$, unless $X \simeq v_{2}\left(\mathbb{P}^{n}\right)$, the Picard group is generated by the hyperplane section and the index of $X$ is at least $\frac{\operatorname{dim}(X)+1}{2}$. Conversely, from a recent result by Bonavero-Höring, see $[\mathrm{BH}]$, it follows that any $n$-dimensional complete intersection which is Fano, of index at least $\frac{n+1}{2}$, is conic-connected; see Proposition 2.4. Moreover, the work of Hwang-Kebekus, see [ $[\mathrm{HK}]$, shows that any Fano $n$-fold of index greater than $\frac{2 n}{3}$ is conic-connected; see Proposition 2.5. Applications of Theorem 2.2 to the classification of secant and dual defective manifolds, special Cremona transformations, etc. may be found in [Ru, IR].

The proof of Theorem 2.2 naturally fits into the context of Mori Theory, as established by the ground-breaking papers [Mo1, Mo2]. Besides the use of such powerful tools as the Cone Theorem, contractions of extremal rays, etc., we also need the very efficient method of algebraizing the equivalence relation associated to a covering family of curves. This technique, pioneered by Campana [Ca], was further developed and successfully applied by Kollár, Miyaoka, and Mori in their seminal paper [KMM]. The recent characterization of projective space, belonging to the same circle of ideas, and due to Cho, Miyaoka, and Shepherd-Barron (see $[\mathrm{CMSB}]$ ), is also used in the proof of Theorem 2.2. Finally, a key ingredient of the argument is our Theorem 1.5, a "global version" of Theorem 1.3 .

Theorem 1.3 which, we hope, has some independent interest, generalizes [IN] Theorem 4.2] (see also [โأ2]). Its content is a criterion of rationality: $X$ is rational if and only if it admits a covering family of rational 1-cycles, all passing through $x$, all smooth at $x$ and such that the general cycle of the family is a curve, uniquely determined by its tangent line at $x$. In Theorem 1.5, under suitable assumptions, the birational isomorphism between $X$ and $\mathbb{P}^{n}$ is shown to be biregular along a general (smooth) curve of the given family; moreover, the general member of the family corresponds to a line in $\mathbb{P}^{n}$.

We acknowledge our intellectual debt to the pioneering work of G. Scorza [Sc1] Sc2], as well as to all the previous papers mentioned above.

We work over $\mathbb{C}$; note however that Theorem 1.3 holds in any characteristic.

\section{A RATIONALITY CRITERION}

Let $X$ be a projective variety. A family of rational 1-cycles on $X, \mathcal{F} \rightarrow \mathcal{C}$, is given by a closed subset $\mathcal{C} \subset \operatorname{Chow}(X)$ such that for each 1-cycle of the family, all its irreducible components are rational. Moreover, we always assume that $\mathcal{C}$ is irreducible and the general member of $\mathcal{C}$ is an irreducible reduced (rational) curve.

Definition 1.1. A covering family (of rational 1-cycles, as above) on $X$ is a family $\mathcal{F} \rightarrow \mathcal{C}$ as above, such that the tautological morphism $\mathcal{F} \rightarrow X$ is surjective.

Let $x \in X$ be a smooth point. 
Definition 1.2. An $x$-covering family on $X$ is a covering family $\mathcal{F} \rightarrow \mathcal{C}$ as above, such that, moreover, $x \in \operatorname{Supp}(C)$ for any $C \in \mathcal{C}$ and the general member of $\mathcal{C}$ is smooth at $x$.

An $x$-covering family is called maximal if $\mathcal{C}$ is an irreducible component of the closed subscheme of $\mathrm{Chow}_{x}(X)$, whose points correspond to rational 1-cycles.

An $x$-covering family is said to be smooth if all its 1-cycles are smooth at $x$.

An $x$-covering family is said to satisfy the infinitesimal uniqueness property if the general member of the family is uniquely determined by its tangent space at $x$.

Recall that $X$ is rationally connected if through any two (general) points of $X$ there passes a rational curve. Saying that $X$ is rationally connected is equivalent to the existence of an $x$-covering family. One can also see that the existence of an $x$-covering family satisfying the infinitesimal uniqueness property is equivalent to $X$ being unirational. The next result gives a criterion of rationality, in the spirit of the papers [IN, Io2]. It may be seen as a birational version of a series of characterizations of $\mathbb{P}^{n}$ in terms of families of rational curves, started in Mori's famous paper [Mo1].

Theorem 1.3. Let $X$ be a projective variety. The following conditions are equivalent:

(i) $X$ is rational;

(ii) for some $x \in X, X$ admits a smooth $x$-covering family satisfying the infinitesimal uniqueness property.

Proof. To see that (i) implies (ii), note that a neighbourhood of a general point $x \in X$ is isomorphic to some neighbourhood of a point $y \in \mathbb{P}^{n}$. Simply consider the family induced on $X$ by the lines of $\mathbb{P}^{n}$ passing through $y$.

(ii) implies (i), the non-trivial implication, is proved in the following three steps.

Step I. The basic diagram.

Let $\pi: \mathcal{F} \rightarrow \mathcal{C}$ be our $x$-covering family and let $\varphi: \mathcal{F} \rightarrow X$ be the tautological morphism.

Note that $\pi$ admits a section $\mathcal{E}$, which is contracted by $\varphi$ to the point $x$. Consider the blowing-up $\sigma: X^{\prime} \rightarrow X$ of $X$ at $x$. Via [IN] Lemma 4.3], the universal property of the blowing-up shows that we have a morphism $\psi: \mathcal{F} \rightarrow X^{\prime}$, such that $\sigma \circ \psi=\varphi$. So we have the following diagram

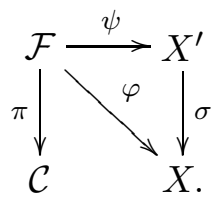

In particular, $\psi$ maps the section $\mathcal{E}$ to $E$, the exceptional locus of $\sigma$. Let $\psi_{0}: \mathcal{E} \rightarrow$ $E$ be the restriction of $\psi$.

Step II. $\psi_{0}$ is generically finite and surjective; in particular, $\operatorname{dim}(\mathcal{C})=n-1$.

This follows from an elementary, but very useful remark, due to Kebekus [Ke1], proof of Theorem 3.4. Indeed, consider a general point $p \in E \simeq \mathbb{P}\left(T_{x}^{*}(X)\right)$ and let 
$C \subset \mathcal{E} \simeq \mathcal{C}$ be a curve such that $\psi_{0}(C)=p$. Take $\widetilde{C}$, the normalization of $C$, and consider the surface $S$ over $\widetilde{C}$ which is got by base-change from our family. Let $D \subset S$ be the induced section for $\pi_{S}: S \rightarrow \widetilde{C}$ and note that $S$ is smooth along $D$ by our hypothesis. The restriction of the tangent morphism of $\varphi_{S}: S \rightarrow X$ induces a morphism $T_{\varphi_{S}}: N_{D \mid S} \rightarrow l_{p} \simeq \mathbb{C}$, where $N_{D \mid S}$ is the (geometric) normal bundle of $D$ in $S$ and $l_{p}$ is the line in $T_{x}(X)$ corresponding to $p \in E$. Since $N_{D \mid S}$ is not trivial, the above map has a zero. The corresponding curve of the family is singular at $x$. This is a contradiction. To conclude, note also that $\operatorname{dim}(\mathcal{F}) \geqslant n$, so $\operatorname{dim}(\mathcal{E}) \geqslant n-1$, while $\operatorname{dim}(E)=n-1$. In particular, $\operatorname{dim}(\mathcal{C})=n-1$ and $\varphi$ (or $\psi$ ) is generically finite.

Step III. Conclusion.

Note first that the infinitesimal uniqueness property of our family translates into saying that the map $\psi_{0}$ is birational. Observe also that we have $\psi^{-1}(E)=\mathcal{E}$. Now, use properness of $\psi$ and the fact that $\psi_{0}$ is birational to deduce that $\psi$ is birational. We have already seen that $\mathcal{C}$ is birational to $E \simeq \mathbb{P}^{n-1}$. Replacing $\mathcal{F}$ by its normalization $\widetilde{\mathcal{F}}$ and using the section $\mathcal{E}$, we find that $\widetilde{\mathcal{F}}$ is generically a $\mathbb{P}^{1}$-bundle over $\mathcal{C}$. Thus $X$ is rational, being birational to $\widetilde{\mathcal{F}}$.

The next result is a "more global" version of Theorem 1.3 , it improves [IN] Theorem 4.2]. In the terminology of [IV], (ii) says that the model $(X, C)$ is equivalent to $\left(\mathbb{P}^{n}\right.$, line $)$. Let us recall the following definition from $[\overline{\mathrm{BBI}}]$ :

Definition 1.4 ([BBBI]). A smooth rational curve $C \subset X$, where $X$ is a projective manifold of dimension $n$, is a quasi-line if $N_{C \mid X} \simeq \bigoplus_{1}^{n-1} \mathcal{O}_{\mathbb{P}^{1}}(1)$.

Theorem 1.5. Let $X$ be a projective manifold and let $\mathcal{C}$ be a smooth maximal $x$-covering family of it. Let $\mathcal{D} \subset \mathcal{C}$ denote the family of reducible cycles in $\mathcal{C}$.

(i) If the general member of $\mathcal{C}$ is smooth, it is a quasi-line (this is always the case if $\operatorname{dim}(X) \geqslant 3$ ).

(ii) Assume moreover that the general member of $\mathcal{C}$ meets the locus of $\mathcal{D}$ only at $x$ (this is true, e.g. if $\operatorname{codim}_{\mathcal{C}}(\mathcal{D}) \geqslant 2$ ). Then, for any quasi-line $C \in \mathcal{C}$, there are a neighbourhood $U$ of $C$ in $X$ and a neighbourhood $V$ of a line $l \subset \mathbb{P}^{n}$, together with an isomorphism $U \simeq V$, sending $C$ to $l$.

Proof. We use the setting and notation from the proof of Theorem 1.3

(i) Let $C \in \mathcal{C}$ be a general smooth curve. As $\mathcal{C}$ is a maximal $x$-covering family, $N_{C \mid X}$ is ample. In Step II from the proof of Theorem 1.3 we saw that $\operatorname{dim}(\mathcal{C})=$ $n-1$, so $C$ is a quasi-line.

(ii) We first prove the following:

Claim: There is a unique quasi-line from our given family passing through a general point of $X$ (i.e. the map $\varphi$ is birational).

Let $D \subset X^{\prime}$ be the locus of points belonging to the images via $\psi$ of reducible cycles from $\mathcal{C}$. By (i), a general curve $C$ from our family $\mathcal{C}$ is a quasi-line in $X$. By the assumption in (ii), if $\tilde{C}$ denotes its proper transform on $X^{\prime}$, we have $\tilde{C} \cap D=\emptyset$ for $C$ general. So any point $c \in \pi\left(\psi^{-1}(\tilde{C})\right)$ represents an irreducible member of 
the family $\mathcal{C}$. Now, the birationality of the map $\varphi$ follows from the proof of [Ko, V.3.7.5.], a result due to Miyaoka. So the Claim is proved.

The Claim allows us to apply [Io2, Theorem 2.5] in order to get the conclusion in (ii). Finally, observe that $\operatorname{codim}_{\mathcal{C}}(\mathcal{D}) \geqslant 2$ implies $\operatorname{codim}_{X^{\prime}}(D) \geqslant 2$. Moreover, if $C$ is a general member of $\mathcal{C}$, the normal bundle of $\tilde{C}$ in $X^{\prime}$ is trivial; see, for instance, [IN] Lemma 1.6]. So, by [Ko, II.3.7], we have $\tilde{C} \cap D=\emptyset$ for $C$ general.

\section{Classification OF CONIC-CONNECTED MANifOldS}

Definition 2.1 (cf. also $[\mathrm{KS}]$ ). A smooth irreducible non-degenerate projective variety $X \subset \mathbb{P}^{N}$ is said to be a conic-connected manifold, briefly a $C C$-manifold, if through two general points of $X$ there passes an irreducible conic contained in $X$.

The following classification theorem is our main result; as CC-manifolds are stable under isomorphic projection, we may assume $X$ to be linearly normal.

Theorem 2.2. Let $X \subset \mathbb{P}^{N}$ be a smooth irreducible linearly normal non-degenerate $C C$-manifold of dimension $n$. Then either $X \subset \mathbb{P}^{N}$ is a Fano manifold with $\operatorname{Pic}(X) \simeq \mathbb{Z}\left\langle\mathcal{O}_{X}(1)\right\rangle$ and of index $i(X) \geqslant \frac{n+1}{2}$, or it is projectively equivalent to one of the following:

(i) $\nu_{2}\left(\mathbb{P}^{n}\right) \subset \mathbb{P}^{\frac{n(n+3)}{2}}$.

(ii) The projection of $\nu_{2}\left(\mathbb{P}^{n}\right)$ from the linear space $\left\langle\nu_{2}\left(\mathbb{P}^{s}\right)\right\rangle$, where $\mathbb{P}^{s} \subset \mathbb{P}^{n}$ is a linear subspace; equivalently $X \simeq \mathrm{Bl}_{\mathbb{P}^{s}}\left(\mathbb{P}^{n}\right)$ embedded in $\mathbb{P}^{N}$ by the linear system of quadric hypersurfaces of $\mathbb{P}^{n}$ passing through $\mathbb{P}^{s}$; alternatively $X \simeq \mathbb{P}_{\mathbb{P} r}(\mathcal{E})$ with $\mathcal{E} \simeq \mathcal{O}_{\mathbb{P} r}(1)^{\oplus n-r} \oplus \mathcal{O}_{\mathbb{P} r}(2), r=1,2, \ldots, n-1$, embedded by $\left|\mathcal{O}_{\mathbb{P}(\mathcal{E})}(1)\right|$. Here $N=\frac{n(n+3)}{2}-\left(\begin{array}{c}s+2 \\ 2\end{array}\right)$ and $s$ is an integer such that $0 \leqslant s \leqslant n-2$.

(iii) A hyperplane section of the Segre embedding $\mathbb{P}^{a} \times \mathbb{P}^{b} \subset \mathbb{P}^{N+1}$. Here $n \geqslant 3$ and $N=a b+a+b-1$, where $a \geqslant 2$ and $b \geqslant 2$ are such that $a+b=n+1$.

(iv) $\mathbb{P}^{a} \times \mathbb{P}^{b} \subset \mathbb{P}^{a b+a+b}$ Segre embedded, where $a, b$ are positive integers such that $a+b=n$.

Corollary 2.3. CC-manifolds are Fano and have second Betti number $b_{2} \leqslant 2$; when $b_{2}=2$, they are also rational.

Proof of Theorem 2.2. Fix a general point $x \in X$. By definition of CC-manifold, there exists a (maximal) $x$-covering family of conics on $X$; we fix one such family, which we denote by $\mathcal{C}_{x}$.

Assume first that there exist lines $l_{1}$ and $l_{2}$ such that $l_{1} \cup l_{2}$ belongs to $\mathcal{C}_{x}$.

Let $[C]$ denote the numerical class in $\operatorname{Num}(X)$ of the curve $C$. Thus, if we let [Q] be the class of a general conic from the family $\mathcal{C}_{x}$, we have $[Q]=\left[l_{1}\right]+\left[l_{2}\right]$. Let $V_{i}, i=1,2$, be the irreducible proper component of $\operatorname{Chow}_{1,1}(X)$ to which $l_{i}$ belongs, notation as in [Ko].

As in the first section, let us call the family $V_{i}$ covering if its curves fill up $X$. Observe that, by the generality of the point $x \in X$, at least one of the families $V_{1}, V_{2}$ is so. 
For each covering family $V_{i}$, define a relation $R_{i}$ on $X$ by saying that two points $x_{1}, x_{2} \in X$ are equivalent if there exists a connected chain of lines from the family $V_{i}$ joining the points $x_{1}$ and $x_{2}$. This is the $\left\langle\left(U_{i}\right)_{1}\right\rangle$-relation, $i=1,2$, for the normal form associated to the proper connected universal family $U_{i} \rightarrow V_{i}[\mathrm{Ko}$, Sections IV.4, IV.4.4 and IV.4.8.3].

Theorem IV.4.16 of [Ko] (see also [De, Theorem 5.9]) yields the existence of an open subset $X_{i}^{0} \subseteq X$ and of a proper morphism with connected fibers $\pi_{i}$ : $X_{i}^{0} \rightarrow Z_{i}^{0}$ such that the $\left\langle\left(U_{i}\right)_{1}\right\rangle$-relation restricts to an equivalence relation on $X_{i}^{0}$ and such that, for every $z \in Z_{i}^{0}, \pi_{i}^{-1}(z)$ coincides with the equivalence relation class defined by $V_{i}, i=1,2$. Since the family is covering the equivalence class of a point in $X_{i}^{0}$ does not reduce to the point itself. Therefore the equivalence class of a general point $x \in X$ is scheme theoretically a smooth irreducible positive dimensional projective algebraic variety $F_{i}^{x}=\pi_{i}^{-1}\left(\pi_{i}(x)\right)$. Let $a_{i}=\operatorname{deg} N_{l_{i} \mid X}$. Since we are assuming $V_{i}$ covering, $a_{i} \geqslant 0$; the locus of lines (from the family determined by $l_{i}$ ) passing through a general point $x$ will be denoted by $C_{i}^{x}$. It is a cone of dimension $a_{i}+1 \geqslant 1$. Clearly, we have $C_{i}^{x} \subseteq F_{i}^{x}$, so $\operatorname{dim}\left(F_{i}^{x}\right) \geqslant a_{i}+1$ and equality holds if and only if $F_{i}^{x}$ is a linear space.

Case I. Assume that $\left[l_{1}\right]=\left[l_{2}\right]$.

Let $[l]=\left[l_{i}\right], i=1,2$, and let $Q \in \mathcal{C}_{x}$ be a general conic. Let $R_{x}$ be the locus of points on $X$ which can be joined to $x$ by a connected chain of lines whose numerical class is [l]. If $R_{x} \subsetneq X$, reasoning as above, we could construct an open subset $X^{0} \subseteq X$ and a proper morphism with connected fibers $\pi: X^{0} \rightarrow Z^{0}$ such that, for every $z \in Z^{0}, \pi^{-1}(z)$ coincides with the equivalence relation class defined by the irreducible proper component of $\operatorname{Chow}_{1,1}(X)$ to which $l$ belongs. From this it would follow the existence of an effective divisor $D$ passing through a general point $z \in X$ and such that $D \cdot l=0$. This would imply $D \cdot Q=2 D \cdot l=0$, which is clearly impossible. In conclusion $R_{x}=X$, so that the Picard number of $X$ is one by [Ko, IV.3.13.3]. In this case, clearly $\operatorname{Pic}(X)=\mathbb{Z}\langle\mathcal{O}(1)\rangle$. Moreover, $Q$ has ample normal bundle, so $2 i(X)=-K_{X} \cdot Q \geqslant n+1$, giving $i(X) \geqslant \frac{n+1}{2}$.

From now on, assume that $\left[l_{1}\right] \neq\left[l_{2}\right]$, so that $V_{1} \neq V_{2}$.

Case II. Assume that both $V_{1}$ and $V_{2}$ are covering. So $a_{i} \geqslant 0, i=1,2$.

Keeping the notation introduced above, let us observe that

$$
\begin{aligned}
\operatorname{dim}\left(F_{1}^{x}\right)+\operatorname{dim}\left(F_{2}^{x}\right) & \geqslant \operatorname{dim}\left(C_{1}^{x}\right)+\operatorname{dim}\left(C_{2}^{x}\right)=a_{1}+1+a_{2}+1 \\
& =-K_{X} \cdot l_{1}-1+\left(-K_{X}\right) \cdot l_{2}-1 \\
& =-K_{X} \cdot Q-2=\operatorname{dim}\left(\mathcal{C}_{x}\right) \geqslant n-1 .
\end{aligned}
$$

Moreover, since $\left[l_{1}\right] \neq\left[l_{2}\right]$, an $R_{1}$-equivalence class and an $R_{2}$-equivalence class cannot intersect along a curve, see [Ko, IV.3.13.3]. Note that we shall use this systematically in what follows.

Assume first that $\operatorname{dim}\left(F_{1}^{x}\right)+\operatorname{dim}\left(F_{2}^{x}\right)=n$. If $y \in X$ is another general point, using the rational maps $\pi_{i}$ and the fact that $\operatorname{dim}\left(F_{1}^{x} \cap F_{2}^{x}\right) \leqslant 0$, we see that $F_{1}^{x} \cap F_{2}^{y} \neq \emptyset$. In particular, any point of $X$ may be joined to $x$ by a connected chain of rational curves from the families $V_{1}, V_{2}$. It follows from [Ko, IV.3.13.3] 
that $N_{1}(X)_{\mathbb{Q}}=\left\langle\left[l_{1}\right],\left[l_{2}\right]\right\rangle$. Moreover, by [BCD, Proposition 1], both rays $\mathbb{R}^{+}\left[l_{i}\right]$ are extremal since $R_{i}$-equivalence classes are equidimensional, for $i=1,2$.

Assume next that $\operatorname{dim}\left(F_{1}^{x}\right)+\operatorname{dim}\left(F_{2}^{x}\right)=n-1$. By (2.1) this gives $a_{1}+a_{2}+2=$ $n-1$. Denote by $E_{i}^{x}$ the (closed) locus of points lying on rational curves from the family $V_{j}$ meeting $F_{i}^{x}$, for $i \neq j$. We have $\operatorname{dim}\left(E_{i}^{x}\right)=a_{1}+a_{2}+2=n-1$, $i=1,2$. It follows from the definition of $E_{i}^{x}$ that $E_{i}^{x} \cdot l_{j}=0$ for $i \neq j$. We must have $E_{i}^{x} \cdot l_{i}>0$; otherwise we get $E_{i}^{x} \cdot Q=0$, which is absurd since $E_{i}^{x}$ is effective and $x$ is general. From this it follows again that any point of $X$ may be joined to $x$ by a connected chain of rational curves from the families $V_{1}, V_{2}$, so that $N_{1}(X)_{\mathbb{Q}}=\left\langle\left[l_{1}\right],\left[l_{2}\right]\right\rangle$. We claim that both rays $\mathbb{R}^{+}\left[l_{1}\right], \mathbb{R}^{+}\left[l_{2}\right]$ are extremal. Take a curve $C \subset X$ and write its class in $N_{1}(X) \mathbb{Q}$ as $[C]=a_{1}\left[l_{1}\right]+a_{2}\left[l_{2}\right], a_{1}, a_{2} \in \mathbb{Q}$. We may assume (up to a permutation of indices) that $a_{1} \geqslant 0$. If $E_{2}^{x} \cdot C \geqslant 0$, it follows $a_{2} \geqslant 0$ and we are done. If not, we must have $C \subset E_{2}^{x}$. But in this case, a variant of "bend and break" shows that we may write $[C]=\alpha_{1}\left[l_{1}\right]+\alpha_{2}\left[l_{2}\right]$, with $\alpha_{2} \geqslant 0$. To the best of our knowledge, this last trick was first noticed in [BSW, Lemma 1.4.5].

In conclusion, the cone of effective 1-cycles $N E(X)=\overline{N E(X)}=\mathbb{R}^{+}\left[l_{1}\right]+$ $\mathbb{R}^{+}\left[l_{2}\right]$ and $-K_{X}$ is ample by the Kleiman criterion. Thus $X$ is a Fano manifold with $\operatorname{Pic}(X) \simeq \mathbb{Z} \oplus \mathbb{Z}$.

There exist two contractions $f_{i}: X \rightarrow Z_{i}$ of the extremal rays $\mathbb{R}^{+}\left[l_{i}\right]$, where $Z_{i}$ is a normal projective variety with $\operatorname{Pic}\left(Z_{i}\right) \simeq \mathbb{Z}$ for $i=1,2$. Write, for simplicity, $F_{i}=F_{i}^{x}$ and $C_{i}=C_{i}^{x}$ for $i=1,2$.

From (2.1) it follows that exactly one of the following subcases (a), (b) or (c) may arise.

(a) $F_{i}=C_{i} \simeq \mathbb{P}^{a_{i}+1}, i=1,2, \operatorname{dim}\left(F_{1}\right)+\operatorname{dim}\left(F_{2}\right)=n, \quad$ so $a_{1}+a_{2}+2=n$,

(b) $F_{i}=C_{i} \simeq \mathbb{P}^{a_{i}+1}, i=1,2, \operatorname{dim}\left(F_{1}\right)+\operatorname{dim}\left(F_{2}\right)=n-1$, so $a_{1}+a_{2}+2=n-1$,

(c) $F_{1}=C_{1} \simeq \mathbb{P}^{a_{1}+1}, C_{2} \subsetneq F_{2}, \operatorname{dim}\left(F_{1}\right)+\operatorname{dim}\left(F_{2}\right)=n, \quad$ so $a_{1}+a_{2}+3=n$.

Subcase (a). The linear spaces $F_{1}, F_{2}$ intersect transversally in a point. It follows that the map $f_{1} \times f_{2}: X \rightarrow Z_{1} \times Z_{2} \simeq \mathbb{P}^{a_{2}+1} \times \mathbb{P}^{a_{1}+1}$ is finite and birational, hence an isomorphism. This leads to case (iv).

Subcase (b). At least one of the contractions $f_{1}, f_{2}$ is equidimensional. Otherwise we may find two fibres $F_{1}, F_{2}$ such that $\operatorname{dim}\left(F_{1} \cap F_{2}\right) \geqslant 1$. Assume that $f_{1}$ is equidimensional. From [Fu1, Lemma 2.12] or [Io1, p. 467], it follows that $f_{1}$ : $X \rightarrow Z_{1}$ is a $\mathbb{P}^{a_{1}+1}$-bundle. In particular, $Z_{1}$ is smooth. At this point, we want to apply the characterization of projective space due to Cho-Miyaoka-ShepherdBarron [CMSB] to see that $Z_{1} \simeq \mathbb{P}^{a_{2}+2}$. As the proof of this important criterion is quite involved, we would like to explain how, due to our particular context, the result follows from the simpler argument in [Ke2]. Note that smoothness of $Z_{1}$ is important for this simplification. Let $Q$ be a conic from our family $\mathcal{C}_{x}$. If $Q=l_{1} \cup l_{2}$ is reducible, we have $f_{1}(Q)=f_{1}\left(l_{2}\right)$ and the restriction $f_{1} \mid l_{2}: l_{2} \rightarrow f_{1}\left(l_{2}\right)$ is an isomorphism. Let now $Q$ be irreducible and let $\Pi$ denote the plane it spans. We claim that $f_{1} \mid Q: Q \rightarrow f_{1}(Q)$ is birational. This is seen by looking at the intersection of the linear spaces $\Pi$ and a fibre $F_{1}$. If $x \in X$ is a very general point, 
consider the family of irreducible reduced rational curves $f_{1 *}(Q)$, where $Q \in \mathcal{C}_{x}$. The argument in [Ke2] applies to this family, yielding that $Z_{1} \simeq \mathbb{P}^{a_{2}+2}$ and $f_{1}(Q)$ is a line.

A general conic $Q$ from our family has ample normal bundle and $\operatorname{deg} N_{Q \mid X}=$ $a_{1}+a_{2}+2=n-1$, so $Q$ is a quasi-line. Now fix a general conic $Q_{0}$ and let $L:=f_{1}\left(Q_{0}\right)$, a line in $Z_{1}=\mathbb{P}^{a_{2}+2}$. Let $T:=f_{1}^{-1}(L)$. Fix also two general points $t, t^{\prime} \in T$. Any conic $Q$ passing through $t, t^{\prime}$ is contained in $T$, because $f_{1}(Q)$ is a line. The standard exact sequence of normal bundles

$$
0 \rightarrow N_{Q \mid T} \rightarrow N_{Q \mid X} \rightarrow N_{T|X| Q} \rightarrow 0
$$

shows that $Q$ is a quasi-line in $T$. Therefore, we may apply [IV] Proposition 4.1] to deduce that (up to a Stein factorization) the only non-constant non-finite morphisms defined on $T$ are $f_{1} \mid T$ and $\sigma: T \rightarrow \mathbb{P}^{a_{1}+2}$, a blowing-up sending $Q$ to a line of $\mathbb{P}^{a_{1}+2}$. Consider now the restriction $r:=f_{2} \mid T: T \rightarrow Z_{2}$. As $\operatorname{dim}(T)=a_{1}+2$, by the above considerations, $r$ is surjective. We claim that $r$ is birational. Otherwise, take two distinct points $t_{1}, t_{2}$ in a general fibre, $F_{2} \cap T$, of $r$. The line $\left\langle t_{1}, t_{2}\right\rangle \subset F_{2}$ meets $T$ in two points, so it is contained in $T$ by the above reasoning. This is absurd since $r$ is generically finite. Moreover, $r$ is not an isomorphism, as $\operatorname{Pic}\left(Z_{2}\right) \simeq \mathbb{Z}$ and $\operatorname{Pic}(T) \simeq \mathbb{Z} \oplus \mathbb{Z}$. Therefore, $r=\sigma$, in particular $Z_{2} \simeq \mathbb{P}^{a_{1}+2}$ and $f_{2}(Q)$ is a line.

To conclude subcase (b), we write the hyperplane section of $X$ as $H=H_{1}+H_{2}$, with $H_{i} \cdot l_{j}=1-\delta_{i j}$. We have seen that $f_{i}^{*}\left(D_{i}\right)=H_{i}$, where $D_{i}$ is the hyperplane divisor of $Z_{i}$ for $i=1,2$. The map $f:=f_{1} \times f_{2}: X \rightarrow \mathbb{P}^{a_{2}+2} \times \mathbb{P}^{a_{1}+2}$ is given by a linear system $\Lambda \subseteq|H|$. Let $X^{\prime}:=f(X)$, which is a divisor on $Z:=\mathbb{P}^{a_{2}+2} \times \mathbb{P}^{a_{1}+2}$, and note that $f: X \rightarrow X^{\prime}$ is finite and birational by construction. Since $H \cdot l_{i}=1$ for $i=1,2$, the general fiber of $f_{i}$ is mapped by $f$ onto a hyperplane of $Z_{j}$, for $i \neq j$. Therefore, the divisor $X^{\prime}$ has type $(1,1)$ in $\operatorname{Pic}(Z)$ and $f$ induces an isomorphism on each fiber of $f_{1}$. Thus $f: X \rightarrow X^{\prime}$ is an isomorphism and we get case (iii) of the theorem.

Subcase (c). In this case, both $f_{1}$ and $f_{2}$ are equidimensional and $\operatorname{dim}\left(Z_{2}\right)=$ $n-a_{2}-2=a_{1}+1=\operatorname{dim}\left(F_{1}\right)$. Using the notation and the reasoning above we get $\operatorname{dim}(T)=a_{1}+2$, so $f_{2} \mid T: T \rightarrow Z_{2}$ cannot be birational. This shows subcase (c) does not happen.

Case III. Assume that the family $V_{1}$ is covering and $V_{2}$ is not.

Now we can also assume that the maximal $x$-covering family $\mathcal{C}_{x}$ is smooth (otherwise we are in Case II for some different covering family $V_{2}$ ), so we may use Theorem 1.5 In particular, the general conic in $\mathcal{C}_{x}$ is a quasi-line.

We consider separately the subcases:

$\mathrm{III}_{1} . C_{1}^{x}$ has codimension 1 in $X$.

$\mathrm{III}_{2} . C_{1}^{x}$ has codimension $\geqslant 2$ in $X$.

Case III $_{1}$. Reasoning as in Case II, we deduce that either $X=F_{1}^{x} \supsetneq C_{1}^{x}$, or $F_{1}^{x}=C_{1}^{x}$ is a linear subspace in $\mathbb{P}^{N}$. In the first case, $\operatorname{Pic}(X)=\mathbb{Z}\langle\mathcal{O}(1)\rangle$ and we would fall in Case I, contrary to our assumption. In the second case, $F_{1}^{x}$ has trivial normal bundle, so that $X \subset \mathbb{P}^{N}$ is easily seen to be a scroll over $\mathbb{P}^{1}$, say 
$\pi: X \rightarrow \mathbb{P}^{1}$, having $F_{1}^{x}$ as general fiber. We may write $X \simeq \mathbb{P}(\mathcal{E})$, with $\mathcal{E}=$ $\pi_{*}\left(\mathcal{O}_{X}(1)\right)$ a rank- $n$ ample vector bundle on $\mathbb{P}^{1}$. Since the general conic $Q \in \mathcal{C}_{x}$ is a quasi-line, $-K_{X} \cdot Q=n+1$. Using the formula for the canonical class of $\mathbb{P}(\mathcal{E})$ and the relation $F_{1}^{x} \cdot Q=1$, we obtain $\operatorname{deg}(\operatorname{det} \mathcal{E})=n+1$. As $\mathcal{E}$ is ample, its splitting-type must be $(1, \ldots, 1,2)$ and we get case (ii) with $r=1$ (or $s=n-2$ ).

Case $\mathrm{III}_{2}$.

Claim: If $Q$ is a general member of $\mathcal{C}_{x}, Q$ meets the locus of degenerate conics only at $x$.

Let $D \subset X$ denote the locus of $V_{2}$. By [ $\mathrm{Ko}$, II.3.7], to prove the Claim it is sufficient to show that $D \cap Q=\emptyset$ when $D$ is a prime divisor. In this case $C_{1}^{x}$ is also irreducible and the locus of degenerate conics is $C_{1}^{x} \cup D$.

The strategy for proving the above Claim is in two steps. First, as in Case II, we show that the rationally connected fibration determined by the covering family $V_{1}$ is a $\mathbb{P}^{a_{1}+1}$-bundle over $\mathbb{P}^{n-a_{1}-1}$. Next, we deduce from this that a general conic through $x$ does not intersect $D$, proving the Claim. We now proceed with the details.

Let $y$ be a general point of $F_{1}^{x} \cap D$. As before, let $C_{2}^{y}$ be the cone of lines from $V_{2}$ passing through $y$. The geometrical description of $D$ yields

$$
\operatorname{dim}\left(C_{1}^{x} \cap D\right)+\operatorname{dim}\left(C_{2}^{y}\right)=\operatorname{dim}(D)=n-1 .
$$

Since

$$
\operatorname{dim}\left(C_{1}^{x} \cap D\right)=\operatorname{dim}\left(C_{1}^{x}\right)-1=-K_{X} \cdot l_{1}-2,
$$

we find

$\operatorname{dim}\left(C_{2}^{y}\right)=n-1+K_{X} \cdot l_{1}+2=-K_{X} \cdot Q+K_{X} \cdot l_{1}=-K_{X} \cdot l_{2} \quad$ for $y \in D$ general.

It follows

$$
\operatorname{dim}\left(C_{2}^{y}\right) \geqslant-K_{X} \cdot l_{2} \quad \text { for any } y \in D .
$$

By generality of $x$ and $y$ we may assume that $\left(F_{1}^{x} \cap D\right) \cap C_{2}^{y}$ meets the smooth locus of $D$. We deduce

$$
\begin{aligned}
0 & \geqslant \operatorname{dim}\left(\left(F_{1}^{x} \cap D\right) \cap C_{2}^{y}\right) \geqslant \operatorname{dim}\left(F_{1}^{x} \cap D\right)+\operatorname{dim}\left(C_{2}^{y}\right)-(n-1) \\
& \geqslant \operatorname{dim}\left(C_{1}^{x} \cap D\right)+\operatorname{dim}\left(C_{2}^{y}\right)-(n-1)=0 .
\end{aligned}
$$

In particular, $\operatorname{dim}\left(F_{1}^{x} \cap D\right)=\operatorname{dim}\left(C_{1}^{x} \cap D\right)$. Thus, $F_{1}^{x}=C_{1}^{x}=\mathbb{P}^{a_{1}+1}, a_{1}=$ $\operatorname{deg}\left(N_{l_{1} \mid X}\right) \geqslant 0$. Next we prove that the rationally connected fibration determined by $V_{1}$ is a $\mathbb{P}^{a_{1}+1}$-bundle over some manifold $Z$. Indeed, let $\pi: X^{0} \rightarrow Z^{0}$ be our fibration, which is generically a $\mathbb{P}^{a_{1}+1}$-bundle by the above. We show that $X^{0}=X$ and $\pi$ is equidimensional. Using the notation in [BCD, Proposition 1], assume that $B \neq \emptyset$. We claim that $B \cap D \neq \emptyset$. Indeed, if $b \in B \backslash D$, by definition of $B$ there exists a line $l_{1} \in V_{1}$ contained in $B$ and containing $b$. Since $D \cdot l_{1}>0$, the last claim follows. By [BCD, Proposition 1], there is some equivalence class $\overline{F_{1}}$ contained in $B$, passing through a point $b \in B \cap D$ and having $\operatorname{dim}\left(\overline{F_{1}}\right)>a_{1}+1$. We deduce, using (2.2)

$$
\operatorname{dim}\left(\overline{F_{1}} \cap C_{2}^{b}\right) \geqslant \operatorname{dim}\left(\overline{F_{1}}\right)+\operatorname{dim}\left(C_{2}^{b}\right)-n>a_{1}+1-K_{X} \cdot l_{2}-n=0 .
$$


This would imply $\left[l_{1}\right]=\left[l_{2}\right]$, a contradiction. So, $B=\emptyset$ and $\pi$ is equidimensional. As in Case II, subcase (b), it follows that $\pi: X \rightarrow Z$ is a $\mathbb{P}^{a_{1}+1}$-bundle and $Z \simeq \mathbb{P}^{n-a_{1}-1}$. Moreover, a general conic through $x$ is sent isomorphically by $\pi$ onto a line in $Z$.

Let us fix $Q$, a general conic through $x$. Set $L:=\pi(Q) \subset \mathbb{P}^{n-a_{1}-1}$ and let $T:=\pi^{-1}(L) \rightarrow L$ be the associated $\mathbb{P}^{a_{1}+1}$-bundle over $\mathbb{P}^{1}$. The conic $Q$ is contained in $T$. Reasoning as in Case II, subcase (b), it is immediate to see that $T$ is a CC-manifold, belonging to the class described in Case $\mathrm{III}_{1}$ above. In particular there is a map $\sigma: T \rightarrow \mathbb{P}^{a_{1}+2}$ which is a blowing-up along a linear $\mathbb{P}^{a_{1}}$. General conics in $T$ are identified via $\sigma$ to lines in $\mathbb{P}^{a_{1}+2}$ and $D \cap T$ is the exceptional divisor of $\sigma$. Thus $Q \cap D=Q \cap(D \cap T)=\emptyset$ and the Claim is finally proved.

By Theorem 1.5 (ii), there exists a birational map $\varphi: \mathbb{P}^{n} \rightarrow X \subset \mathbb{P}^{N}$ given by a linear system $\Lambda \subseteq\left|\mathcal{O}_{\mathbb{P}^{n}}(2)\right|$, inducing an isomorphism between a neighbourhood of a line in $\mathbb{P}^{n}$ and a neighbourhood of a conic $Q \in \mathcal{C}_{x}$. In particular $\varphi$ is an isomorphism outside the base locus of $\Lambda$, which we denote by $Y$. We claim that, if $Y \neq \emptyset, Y$ is a linear space $\mathbb{P}^{s}$ with $0 \leqslant s \leqslant n-3$.

Suppose there exist two distinct points $y_{1}, y_{2} \in Y$. The linear system of quadric hypersurfaces $\Lambda$ cannot separate points along the line $\left\langle y_{1}, y_{2}\right\rangle$. Therefore $\left\langle y_{1}, y_{2}\right\rangle \subseteq$ $Y$. The infinitesimal version of the same argument shows $Y$ is reduced, so it is a linear space. Thus we are in one of the cases described in (ii).

Finally, if all conics in $\mathcal{C}_{x}$ are irreducible, we get case (i) of the theorem. This follows directly from Theorem 1.5 (ii).

Conversely, from the recent paper $[\overline{\mathrm{BH}}]$, we see that the condition $i \geqslant \frac{n+1}{2}$ in Theorem 2.2 is optimal.

Proposition 2.4. Let $X \subset \mathbb{P}^{n+r}$ be a smooth non-degenerate complete intersection of dimension $n$ and multi-degree $\left(d_{1}, d_{2}, \ldots, d_{r}\right)$. If we assume that $n \geqslant 2 \sum_{1}^{r} d_{i}-$ $2 r-1$, then $X$ is a CC-manifold.

Proof. Note that the inequality in the statement means that $X$ is Fano, of index at least $\frac{n+1}{2}$. Replacing $X$ by a general hyperplane section, we may assume that equality holds. Therefore, we may apply [ $[\mathrm{BH}$, Corollary 1.2] to conclude.

The next result, essentially due to Hwang-Kebekus [HK, Theorem 3.14], shows that any Fano manifold of high index is conic-connected. Note that we slightly improve the bound on the index given in $[\mathrm{HK}]$. The first part of the proposition is well known.

Proposition 2.5 (cf. $[\overline{\mathrm{HK}}]$ ). Let $X \subset \mathbb{P}^{N}$ be a Fano manifold with $\operatorname{Pic}(X) \simeq$ $\mathbb{Z}\langle H\rangle$ and $-K_{X}=i(X) H, H$ being the hyperplane section and $i(X)$ the index of $X$.

(i) If $i(X)>\frac{n+1}{2}$, then $X \subset \mathbb{P}^{N}$ is ruled by lines and for general $x \in X$ the Hilbert scheme of lines through $x, Y_{x} \subset \mathbb{P}\left(T_{x}^{*}(X)\right)=\mathbb{P}^{n-1}$, is smooth. If $i(X) \geqslant \frac{n+3}{2}, Y_{x}$ is also irreducible. Let $S Y_{x}$ be the secant variety of $Y_{x}$ in $\mathbb{P}^{n-1}$

(ii) If $i(X) \geqslant \frac{n+3}{2}$ and $S Y_{x}=\mathbb{P}^{n-1}$, then $X \subset \mathbb{P}^{N}$ is a CC-manifold. 
(iii) If $i(X)>\frac{2 n}{3}$, then $X \subset \mathbb{P}^{N}$ is a CC-manifold.

Proof. By a theorem of Mori [Mo1] the variety $X \subset \mathbb{P}^{N}$ is ruled by a family of rational curves $\mathcal{L}$ such that for $L \in \mathcal{L}$ we have $-K_{X} \cdot L \leqslant n+1$. It follows that $X \subset \mathbb{P}^{N}$ is ruled by lines, that is $H \cdot L=1$. As is well known, see [Ko, II.3.11.5] for a more general result, the Hilbert scheme of lines passing through $x$ is smooth equidimensional and may be identified with a subscheme $Y_{x} \subset \mathbb{P}\left(T_{x}^{*}(X)\right)=\mathbb{P}^{n-1}$ of dimension $i(X)-2$. Thus, if $i(X)-2 \geqslant \frac{n-1}{2}, Y_{x} \subset \mathbb{P}^{n-1}$ is irreducible.

Part (ii) follows from [HK, Theorem 3.14].

To prove part (iii), first observe that $i(X) \geqslant \frac{n+3}{2}$, unless $n \leqslant 6$. If $n \leqslant 6$, $i(X)>\frac{2 n}{3}$ gives $i(X) \geqslant n-1$, so the conclusion follows by the classification of del Pezzo manifolds (see [ [Fu2]). To conclude, using part (ii), it is enough to see that $S Y_{x}=\mathbb{P}^{n-1}$.

By [ $\mathrm{Hw}$, Theorem 2.5], the variety $Y_{x} \subset \mathbb{P}^{n-1}$ is non-degenerate and by hypothesis $n-1<\frac{3 i(X)-6}{2}+2=\frac{3 \operatorname{dim}\left(Y_{x}\right)}{2}+2$, so that $S Y_{x}=\mathbb{P}^{n-1}$ by Zak Linear Normality Theorem; see [Za, V.1.13].

\section{ACKNOWLEDGEMENTS}

Both authors are grateful to the organizers of the Conference "Projective varieties with unexpected properties", that took place in Siena, between 8-13 of June 2004. Our collaboration started while taking part in this very pleasant and fruitful mathematical event.

We thank Tommaso de Fernex and Ciro Ciliberto for a critical reading of previous versions of our paper.

\section{REFERENCES}

[BBI] L. BǍdescu, M.C. Beltrametti, P. IonesCU, Almost-lines and quasi-lines on projective manifolds, in Complex Analysis and Algebraic Geometry (in memory of Michael Schneider), de Gruyter, 2000, pp. 1-27.

[BSW] M.C. Beltrametti, A.J. Sommese, J. Wiśniewski, Results on varieties with many lines and their applications to adjunction theory, in Complex Algebraic Varieties (Bayreuth, 1990), Lecture Notes in Math., 1507, Springer, 1992, pp. 16-38.

[BCD] L. Bonavero, C. Casagrande, S. Druel, On covering and quasi-unsplit families of curves, J. Eur. Math. Soc. 9 (2007), 45-57.

[BH] L. BONAVERo, A. HöRING, Counting conics in complete intersections, Preprint (2008), math. AG/0804162.

[Ca] F. CAMPANA, Coréduction algébrique d'un espace analytique faiblement kählérien compact, Invent. Math. 63 (1981), 187-223.

[CMSB] K. Cho, Y. MiYAoKA, N. Shepherd-BARron, Characterizations of projective space and applications to complex symplectic manifolds, in Higher Dimensional Birational Geometry (Kyoto, 1997), Adv. Stud. Pure Math., vol. 35, Math. Soc. Japan, 2002, pp. 1-88.

[De] O. DebarRe, Higher-Dimensional Algebraic Geometry, Universitext, Springer, 2001.

[Fu1] T. FUJITA, On polarized manifolds whose adjoint bundles are not semipositive, in Algebraic Geometry (Sendai, 1985), Adv. Stud. Pure Math., vol. 10, North-Holland, 1987, pp. 167178.

[Fu2] T. Fujita, Classification Theories of Polarized Varieties, London Math. Soc. Lecture Note Ser., vol. 155, Cambridge Univ. Press, 1990. 
[Hw] J.M. HwANG, Geometry of minimal rational curves on Fano manifolds, in School on Vanishing Theorems and Effective Results in Algebraic Geometry (Trieste, 2000), ICTP Lect. Notes, vol. 6, Abdus Salam Int. Cent. Theoret. Phys., 2001, pp. 335-393.

[HK] J.M. Hwang, S. Kebekus, Geometry of chains of minimal rational curves, J. Reine Angew. Math. 584 (2005), 173-194.

[Io1] P. Ionescu, Generalized adjunction and applications, Math. Proc. Cambridge Philos. Soc. 99 (1986), 457-472.

[Io2] P. IONESCU, Birational geometry of rationally connected manifolds via quasi-lines, in Projective Varieties with Unexpected Properties (Siena, 2004), de Gruyter, 2005, pp. 317-335.

[IN] P. IONESCU, D. NAIE, Rationality properties of manifolds containing quasi-lines, Internat. J. Math. 14 (2003), 1053-1080.

[IR] P. IONESCU, F. Russo, Varieties with quadratic entry locus. II, Compositio Math. 144 (2008), 949-962.

[IV] P. IONESCU, C. VoICA, Models of rationally connected manifolds, J. Math. Soc. Japan 55 (2003), 143-164.

[Ke1] S. KebeKUS, Families of singular rational curves, J. Algebraic Geom. 11 (2002), 245-256.

[Ke2] S. KebEKUS, Characterizing the projective space after Cho, Miyaoka and Shepherd-Barron, in Complex Geometry (Göttingen, 2000), Springer, 2002, pp. 147-155.

[Ko] J. KolláR, Rational Curves on Algebraic Varieties, Ergeb. Math. Grenzgeb. (3), vol. 32, Springer, 1996.

[KMM] J. Kollár, Y. Miyaoka, S. Mori, Rationally connected varieties, J. Algebraic Geom. 1 (1992), 429-448.

[KS] Y. KACHI, E. SATO, Segre's Reflexivity and an Inductive Characterization of Hyperquadrics, Mem. Amer. Math. Soc., vol. 160, no. 763, 2002.

[Mo1] S. MORI, Projective manifolds with ample tangent bundle, Ann. of Math. 110 (1979), 593606.

[Mo2] S. MoRI, Threefolds whose canonical bundles are not numerically effective, Ann. of Math. 116 (1982), 133-176.

[Ru] F. Russo, Varieties with quadratic entry locus. I, Preprint (2006), math. AG/0701889, to appear in Math. Ann.

[Sc1] G. ScorzA, Sulla determinazione delle varietà a tre dimensioni di $S_{r}(r \geqslant 7)$ i cui $S_{3}$ tangenti si tagliano a due a due, Rend. Circ. Mat. Palermo 25 (1908), 193-204; contained in Opere Scelte, vol. I, Ed. Cremonese, 1960, pp. 167-182.

[Sc2] G. SCORZA, Sulle varietà a quattro dimensioni di $S_{r}(r \geqslant 9)$ i cui $S_{4}$ tangenti si tagliano a due a due, Rend. Circ. Mat. Palermo 27 (1909), 148-178; contained in Opere Scelte, vol. I, Ed. Cremonese, 1960, pp. 252-292.

[Za] F.L. ZAK, Tangents and Secants of Algebraic Varieties, Transl. Math. Monogr., vol. 127, Amer. Math. Soc., 1993.

University of Bucharest, Faculty of Mathematics And Computer SCIEnce, 14 ACADEMIEI STR., RO-010014 BUCHAREST, AND InSTITUTE OF MATHEMATICS OF THE RoMANIAN ACADEMY, P.O. BOX 1-764, RO-014700 BUCHAREST, ROMANIA

E-mail address: Paltin. Ionescu@imar.ro

DiPARTIMENTO Di MATEMATICA E INFORMATICA, UNIVERSITÀ DEGLI STUDI DI CATANIA, Viale A. Doria, 6, 95125 CATANia, ITALy

E-mail address: frusso@dmi.unict.it 